\begin{tabular}{|l|l|r|l|}
\hline Eiszeitalter u. Gegenwart & 34 & $\begin{array}{c}131-153 \\
14 \mathrm{Abb} ., 1 \mathrm{Tab} .\end{array}$ & Hannover 1984 \\
\hline
\end{tabular}

\title{
Insektenfraßspuren in letztinterglazialen Hölzern aus den Sanden und Kiesen der nördlichen Oberrheinebene
}

\author{
RALF KLINGER \& WighaRt VON KoENIGSWALD \\ mit einem Beitrag von ANGELA KreUZ
}

Fossil wood, nutrition traces, Hymenopteroida, Coleopteroida, parasitism, Upper Pleistocene, Eemian, paleoecology, forest, river floodplain, gravel, sand.

Upper Rhine Valley, Hessen

TK 25 Nr.: 6116, 6316

Kurzfassung: Aus den jungpleistozänen Schottern der Oberrheinebene werden Hölzer des letzten Interglazials beschrieben, die von Holzinsekten befallen sind. An Hölzern von Picea vel Larix $(3 \mathrm{x})$, Abies alba $(2 \mathrm{x})$, Ulmus sp. $(2 \mathrm{x})$ und Fraxinus excelsior $(1 \mathrm{x})$ werden Vertreter der Siricidae (Holzwespen) [Hymenoptera], der Anobiidae (Klopfkäfer), Buprestidae (Prachtkäfer), Cerambycidae (Bockkäfer), Curculionidae (Bohrrüsselkäfer) und Scolytidae (Borkenkäfer) [Coleoptera], an ihren Fraßgängen nachgewiesen. Im Falle des Prachtkäfers ist die Gruppe auch durch mehere Larvenfunde gesichert. Belegt sind weiterhin eine Parasitierung durch Braconidae vel Bethylidae vel Chalcididae, Hymenoptera, sowie eine Prädation durch Spechte und schließlich eine sekundäre Nutzung von Holzkäfergängen durch Grabwespen (Sphecidae, Hymenoptera). In dieser Insektenfauna wird ein palökologisch aussagefähiger Teil der Lebensgemeinschaft des interglazialen Auewaldes erfaßt.

\section{[Insect Larval Tunnels in Wood of the Last Interglacial from the Oberrheinebene, W.-Germany]}

A bstract: Last interglacial assemblages of fossil wood, attacked by insects, are described from the upper pleistocene gravels in the Oberrheinebene. In the wood of Picea vel Larix ( $3 \mathrm{x})$, Abies alba $(2 \mathrm{x})$, Ulmus sp. $(2 \mathrm{x})$ und Fraxinus excelsior $(1 \mathrm{x})$ there are identified by their larval tunnels a member of the woodwasps (Siricidae, Hymenoptera) as well as members of the furniture beetles (Anobiidae), a member of the wood borers (Buprestidae), which is confirmed by the finding of several fossilisized larvals, members of the longhornbeetles (Cerambycidae), and a member of weevils (Curculionidae) and ambrosia beetles (Scolytidae, Coleoptera) Futhermore there is proved a case of parasitism by wasps (Braconidae vel Bethylidae vel Chalcididae), a case of predation by woodpeckers and a case of reutilisation of larval insecttunnels in wood by a fossorial wasp (Sphecidae, Hymenoptera). This insect fauna seizes a part of the biome of palaeoecological value of a last interglacial forest of river floodplains.

Anschrift der Autoren: Dr. R. Klinger und Dr. W. v. Koenigswald, Zoologische und Geologische Abteilungen des Hessischen Landesmuseums, Friedensplatz 1, D-6100 Darmstadt. Dipl. Biol. A. Kreuz, Seminar für Vor- und Frühgeschichte, Arndtstr. 11, D-6000 Frankfurt a.M. 


\section{Inhaltsverzeichnis}

1. Einleitung

2. Material und Methoden

3. Bestimmung der Holzproben mit Insektefraßspuren (A. KREUZ)

4. Die Altersstellung der Hölzer

5. Beschreibung der Fraßgänge und Bestimmung der Insekten

5.1 Methoden

5.2 Befunde an Hölzern von Picea/Larix (RSH 20, RSH 21, RSH 28)

5.3 Befunde an Hölzern von Abies (RSH 23, RSH 24)

5.4 Befunde an Hölzern von Ulmus (RSH 22, RSH 26)

5.5 Befunde an Hölzern von Fraxinus (RSH 25)

6. Biologisches Umfeld der Holzinsekten

7. Klimatischer Aussagewert der Holzinsekten

8. Danksagung

9. Schriftenverzeichnis

\section{Einleitung}

Die jungpleistozänen Ablagerungen von Rhein und Neckar in der nördlichen Oberrheinebene werden im Rahmen des vom Bundesministerium für Forschung und Technologie geförderten Projektes „,Terrestrische Paläoklimatologie“ intensiver untersucht. Eine Arbeitsgruppe „Paläoklima am Oberrhein“ hat sich zum Ziel gesetzt, palökologische Daten für das letzte Interglazial und das beginnende Glazial zusammenzutragen. Zu diesem Zweck werden Kiesbaggereien im Raum zwischen Mainz, Darmstadt und Worms regelmäßig begangen und tierische und pflanzliche Fossilien geboren. Neben Knochen und Zähnen der jungpleistozänen Großsäuger werden zahlreiche Hölzer ausgebaggert, die 14 verschiedene Gattungen zuzuordnen sind.

Die ersten Fraßspuren von Insekten an dem fossilen Holz und den jungpleistozänen Rheinsanden wurden im Juli 1982 bei einer gemeinsamen Geländebegehung der Autoren mit Prof. Dr. K. U. LeISTIKOw, Frankfurt, von einem von uns (R. KLINGER) erkannt. Einmal darauf aufmerksam geworden, haben dann Dr. D. SCHweIsS und W.v. KoENIGSWALD bei der kontinuierlichen Überwachung der Kiesgruben weitere von Insekten befallene Hölzer gefunden.

Von den Fraßspuren soll auf die Insekten zurückgeschlossen werden, die selber nur in Ausnahmefällen überliefet sind. In einem Holz wurden körperlich erhaltene Spinnenreste gefunden, die von Grabwespen als Nahrung für die Larven eingebracht wurden. Damit läßt sich im Einzelfall das komplexe biologische Umfeld rekonstruieren.

Hier werden die ersten Befunde an Hölzern mitgeteilt, um auf diese ungewöhnliche Überlieferung paläontologisch wichtiger Daten hinzuweisen.

\section{Material}

Die Bergung der Hölzer ist leider nur im Rahmen der technischen Gewinnung der Sande und Kiese möglich. Auf den Fangrosten der Bagger bleiben größere Holzstücke hängen und werden bei der Entleerung der Roste zur Seite gelegt, wo sie dann aufgelesen und auf Insekten-Fraßspuren hin untersucht werden konnten. Die Art der Förderung von Sand 


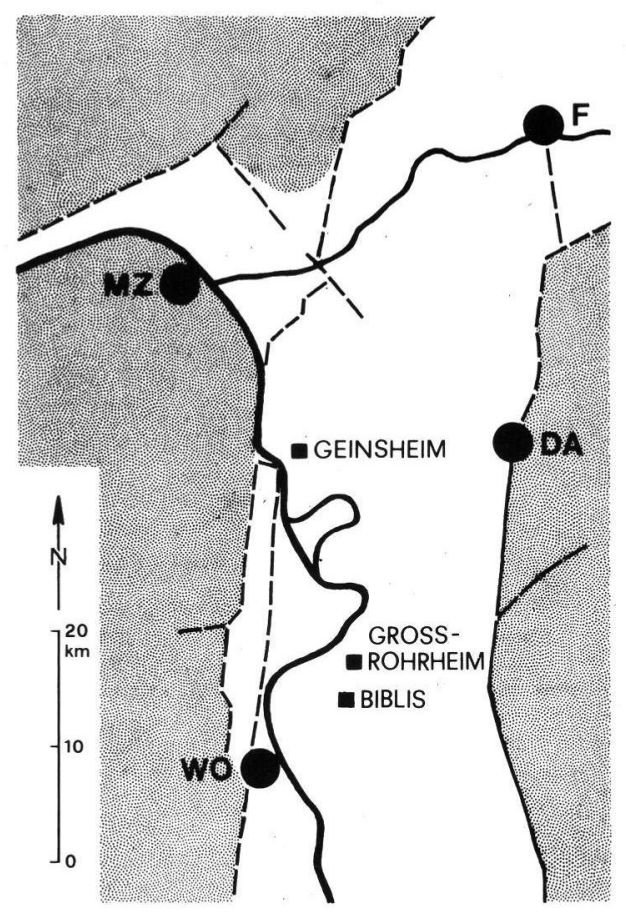

Abb. 1: Lage der Fundorte Geinsheim, Groß-Rohrheim und Biblis in der nördlichen Oberrheinebene, die von Holzinsekten befallene Stamm- und Aststücke aus dem letzten Interglazial geliefert haben.

und Kies führt zu einer recht starken Fraktionierung der im Sediment eingebetteten Hölzer. Wenn das Holz längere Zeit auf den Baggern gelegen hat, ist die Fördertiefe unsicher, ebenso, wenn Material in den Fördertrichter aus dem Hangenden nachgebrochen ist. Bei der folgenden Aufstellung der Funde, nach Fundorten geordnet, werden nur Tiefen angegeben, die als relativ sicher anzusehen sind.

Im einzelnen wurde an folgenden Hölzern ein Befall durch Holzinsekten festgestellt:

Biblis, Grube Heckmann

TK 50, 6316 Worms R 3459600, H 5504930

RSH 20: Flaches Holzstück von Picea/Larix aus dem äußeren Holzkörper aber ohne Bast, Krümmung läßt auf einen Stammdurchmesser von etwa $100 \mathrm{~cm}$ schließen. $(21.7 .1982-$ F $1982-8)$

RSH 21: Rundliches Bruchstück von Picea/Larix, das von einem etwa $40 \mathrm{~cm}$ starken Stamm herrührt. (21.7.1982 - F $1982-8)$ 
Groß-Rohrheim, Grube KBC

TK 50, 6316 Worms R 3460400, H 5507450

RSH 22: Holz von Ulmus sp., Sektor eines Astes oder Stammes, dessen Durchmesser aus der Krümmung der Jahresringe nicht sicher zu erschließen ist, mindestens jedoch $30 \mathrm{~cm}$ betragen hat $(12.8 .1982)$

RSH 23: Zylinderförmisches Holzstück von Abies alba aus dem Inneren eines Astes oder dünnen Stammes. Der vorliegende Teil belegt einen Durchmesser von $14 \mathrm{~cm}$. (9.9.1982 - F $1982-14)$

RSH 24: Sektor aus einem Ast oder Stamm von mindestens $40 \mathrm{~cm}$ Durchmesser von Abies alba. Das Holz stammt aus etwa 18 m unter Flur. (26.1.1983 - F 1983 - 7)

RSH 26: Schmaler Sektor eines größeren Stammes von Ulmus von mindestens $80 \mathrm{~cm}$ Durchmesser. Der Stamm wurde etwa in $19 \mathrm{~m}$ unter Flur gefunden. (7.12.1983 - F 1984 - 9)

RSH 28: Ein $45 \mathrm{~cm}$ langer Sektor aus dem mindestens $35 \mathrm{~cm}$ starken, stark verrotteten Stamm von Picea/Larix. Das Holz wurde in etwa 20-21 m unter Flur geborgen (6.1.1984)

Geinsheim, Grube Kiebert

TK 50, 6116 Darmstadt West, R 3456150, H 5526480

RSH 25: Ein etwa $30 \mathrm{~cm}$ langes Stück eines etwa $15 \mathrm{~cm}$ dicken Astes von Fraxinus excelsior.

Das Holzstück wurde in etwa 20-21 m Tiefe unter Flur ausgebaggert. (2.11.1983 - F 1984 - 5)

Das Belegmaterial wird in der Geol. Abt. des Hessischen Landesmuseums Darmstadt aufbewahrt. Die Nummern RSH 20 etc. beziehen sich auf den Katalog der Rheinschotterfossilien (Holz). Neben dem Funddatum wird mit z.B.: F 1982-8 die Eingangsnummer angegeben.

\section{Bestimmung der Holzproben mit Insektenfraßspuren (ANGELA KREUZ}

Neun Holzproben von drei Fundorten ließen sich nach holzanatomischen Merkmalen bzw. deren Entsprechung rezenter Baumarten in der üblichen Weise (SCHWEINGRUBER 1978) bestimmen:
Biblis
2 Picea/Larix
Geinsheim
1 Fraxinus excelsior
Groß-Rohrheim
2 Abies alba
1 Picea/Larix (mit eingewachsener Wurzel von Clematis vitalba)
3 Ulmus sp.

Kennzeichnende holzanatomische Merkmale der Weißtanne, Abies alba MILL. (Zitation der Pflanzenarten nach FITSCHEN 1983), bieten insbesondere die Markstrahlen mit ihren dicken, teilweise gezähnelten Wänden, ferner fehlen ihr Harzkanäle.

Picea abies (L.) KARST., Gemeine Fichte, und Larix decidua MILL., Europäische Lärche, sind wegen der Übereinstimmung in der Merkmalskombination im Einzelfall holzanatomisch nicht zu trennen. Die Fichte hat im Gegensatz zur Lärche nur selten doppelte Hoftüpfelreihen an den radialen Tracheidenwänden und der Frühholz/Spätholz-Übergang ist kontinuierlich, jedoch sind diese Merkmale - insbesondere bei Berücksichtigung des Erhaltungszustandes des Probenmaterials, sowie möglicher Variationsbreiten der Arten - nicht als trennend $\mathrm{zu}$ werten. 
Ausgehend von der heutigen Verbreitung der beiden Arten könnte Larix decidua für das letzte Interglazial immerhin als weniger wahrscheinlich angenommen werden, da ihr natürliches Vorkommen in Mitteleuropa in den Alpen liegt. Für den Fall aber, daß neben den Ablagerungen des letzten Interglazials, Interstadiale des Früh-Weichsel-Glazials durch Holz belegt sind, ist zu bedenken, daß neben Picea abies auch Picea omorika (PAUE) PURK., Serbische Fichte, vom Alpennordrand bis Norddeutschland vorgekommen ist.

Während dieser Interstadiale, besonders während des Brörup, war Larix auch in den Ebenen zwischen Alpen und Nordsee verbreitet. Die holzanatomische Unterscheidung von Picea abies und P. omorika (VAN DER BURGH, 1978 und GREGUSS, 1955) besonders nach der Dicke der Markstrahlparenchymwände und der Größe der Kreuzungsfeldtüpfel erscheint mir bei dem vorliegenden Material nicht möglich, zumal die Angaben der genannten Autoren erheblich differieren.

Unter Annahme der zeitlichen Einordnung der Proben in ein Interstadial der letzten Eiszeit kann somit auf Grund der holzanatomischen Untersuchung nur die Zuweisung Picea abies/P. omorika/Larix decidua erfolgen. Die Gemeine Esche, Fraxinus excelsior L., hat ein ringporiges Holz. Die auffallend dickwandigen Gefäße im Spätholz sind einzeln oder zu zweien angeordnet, die Markstrahlen schmal, meist zwei- bis dreireihig und homogen.

Wegen der Übereinstimmung der Kombination ihrer holzanatomischen Merkmale sind Ulmus carpinifolia Gled., Feld-Ulme, U. glabra Huds., Berg-Ulme, sowie U. laevis PALL., Flatter-Ulme nicht zu unterscheiden. Allen drei Arten gemeinsam ist ein ringporiges Holz, die Spätholzgefäße sind in zwei- bis vierreihigen mehr oder weniger langen tangentialen bis leicht schrägen Bändern angeordnet, die Markstrahlen meist vier- bis fünfreihig.

Pflanzengeographisch/ökologisch und aktualistisch lassen sich die aufgefundenen Sippen kurz wie folgt chrakterisieren (HEGI 1958, MeUSEL et al. 1965): Die Weißtanne ist in Süd- und Mitteleuropa von tiefen bis in montane Lagen verbreitet, wo sie oft einzeln oder zusammen mit der Fichte oder Buche Bestände bildet. Allgemein genügt ihr ein mäßigwarmes Klima.

Die Fichte ist in Europa vorwiegend in hochmontan-subalpinen Lagen oder temperatboreal verbreitet und bevorzugt ein kühles Klima. Die Lärche ist in den Alpen, Karpaten und im Hügelland von Süd-Polen anzutreffen. In den Alpen bildet sie oft die obere Waldgrenze.

Die Esche, vorwiegend in Auewäldern, reichen Laubwäldern und Gebüschen fast ganz Europas - besonders Mitteleuropas - verbreitet, meidet lichtarme Standorte und rauhe Gebirgslagen und bevorzugt humides, mäßigwarmes Klima. Sie ist jung spätfrostempfindlich und allgemein nicht frosthart, ihr Schwergewicht liegt in submontan-temperaten Bereichen.

Die Ulmenarten sind in Süd- und Mitteleuropa verbreitet (die Feld-Ulme in Mittel- und Südosteuropa), besonders in Auewäldern, Waldrändern und Gebüschen.

U. carpinifolia gehört zu den wärmebedürftigsten einheimischen Holzarten und tritt daher vor allem in tiefer gelegenen, milden Gegenden auf. Im Gebirge wird sie durch die viel weniger empfindliche $U$. glabra ersetzt. Diese steigt z.B. in den Voralpen bis auf $1300 \mathrm{~m}$, erträgt allgemein ein kühl-humides Klima und ist frosthart. U. laevis toleriert feuchte bis nasse Standorte (Überschwemmungszeiger) und bevorzugt ein mäßigwarmes bis warmes Klima. 


\section{Das Alter der Hölzer}

Der nördliche Oberrheingraben ist während des ganzen Pleistozäns ein Senkungsgebiet, das große Mengen fluviatiler Sedimente von Rhein und Neckar aufgenommen hat. Nach BARTZ (1974) erreicht das Quartär bei Heidelberg eine Mächtigkeit von etwa $350 \mathrm{~m}$. Die Kiesgruben, aus denen die hier beschriebenen Hölzer mit Insekten-Fraßspuren stammen, liegen im Dreieck zwischen Darmstadt, Worms und Mainz. Die geologische Gliederung in diesem Raum scheint recht einheitlich zu sein, auch wenn im W geringere Mächtigkeiten der einzelnen Schichtglieder zu erwarten sind als im E.

Unter dem holozänen bzw. jungpleistozänen Hochflutlehm liegt nach SCHEER (1978) die t6-Niederterrasse, die aufgrund absoluter Daten, aber auch des Artenreichtums der kaltzeitlichen Fauna (v. KoENIGSWALD 1983), in das letzte Glazial aber vor dessen Maximum zu stellen ist. Eingetieft in diese Terrasse ist in Rhein-Nähe z.B. in Geinsheim eine t7-Terrasse, die nach absoluten Daten (SCHEER 1978: 285) in das Hoch- und Spätglazial der letzten Vereisung gehört. In Geinsheim wird die t7-Terrasse in einer Tiefe von rund $10 \mathrm{~m}$ von der kaltzeitlichen t6-Terrasse unterlagert.

Lithologisch ist keine scharfe Untergrenze der t6-Niederterrassenschotter zu erkennen. Auffallend ist jedoch, daß in einer Tiefe zwischen 15 und $20 \mathrm{~m}$ recht dicke Baumstämme angetroffen werden. Unter ihnen zeigen bis zu $1 \mathrm{~m}$ dicke, schwarz verwitterte Eichen ein deutlich interglaziales Milieu an. Bei umfangreichen Probenahmen konnten von A. KREUZ im Untersuchungsgebiet bisher 14 Taxa bestimmt werden, darunter Pinus sylvestris/mugo, Picea/Larix, Ulmus sp., Alnus sp., Quercus robur/petraea, Fraxinus excelsior aber auch Taxus baccata, Hippophae rhamnoides und Clematis vitalba.

In der Grube KBC nahe Groß-Rohrheim, aus der die Mehrzahl der hier zu beschreibenden Hölzer mit Insekten-Fraßspuren stammen, sind von A. KREUZ folgende Taxa nachgewiesen worden:

Abies alba MiLl.

Picea abies (L.) KARST/Larix

decidua Mill.

Pinus sylvestries L./ mugo TURRA

Betula pendula ROTH/pubescens EGRH.

Carpinus betulus L.

Fraxinus excelsior $\mathrm{L}$.

Clematis vitalba $\mathrm{L}$.

Pomoideae (Pyrus L./ Malus MiLl./

Crataegus L.)

Quercus robur L./ petraea (MATT.) LIEBL.

Ulmus carpinifolia GLED./glabra Huds./laevis PALL.

\author{
Weißtanne \\ Gemeine Fichte / \\ Europäische Lärche \\ Föhre/Berg-Kiefer \\ Gemeine Birke/Moor-B. \\ Gemeine Hainbuche \\ Gemeine Esche \\ Waldrebe \\ Kernobstgewächse \\ (Birne/Apfel/Weißdorn) \\ Stiel-/Trauben-Eiche \\ Feld-/Berg-/Flatter-Ulme
}

Auch die Fauna dieses Profilabschnittes ist durch warmzeitliche Säugetierarten gekennzeichnet. Als Beispiel soll hier die Liste der Großsäuger aus derselben Kiesgrube bei GroßRohrheim gegeben werden.

Panthera leo speleaea (GOLDFUss) Elephas (Palaeoloxodon) antiquus

(FALCONER \& CAUTLEY)

Mammuthus primigenius (BLUMENBACH)

Equus sp.

Dicerorhinus kirchbergensis (JäGER)
Höhlenlöwe

Waldelefant

Mammut

Pferd

Merck'sches Nashorn 
Sus scrofa L.

Hippopotamus amphibius L.

Capreolus capreolus (L.)

Megaloceros giganteus (BLUMENBACH)

Cervus dama L.

Cervus elaphus L.

Bison priscus (BOJANUs)

Bos vel Bison
Wildschwein

Flußpferd

Reh

Riesenhirsch

Damhirsch

Rothirsch

Bison

großes Rind

Diese Liste enthält die typischen Elemente einer Interglazialfauna. VON KOENIGSWALD \& LÖSCHER (1982) haben deutlich gemacht, daß es sich bei dem Profilabschnitt mit Eichenhölzern aus geologischen Gründen nur um das letzte Interglazial handeln kann. Die Häufigkeit und die Erhaltung der Hippopotamus-Funde schließt eine Umlagerung aus Sedimenten eines älteren Interglazials aus. Dagegen muß damit gerechnet werden, daß manche Einzelfunde der Fauna aus dem Hangenden, das heißt aus Ablagerungen des letzten Glazials stammen. Das gilt für Funde von Mammuthus, Equus, Megaloceros und Bison, wenn auch keineswegs für alle Belege dieser Gattungen.

Der Nachweis von Hippopotamus amphibius in der Eichenholz führenden Schicht weist auf Temperaturen hin, die zumindest in den Wintermonaten höher lagen, als sie derzeit im nördlichen Oberrheingebiet herrschen. Damit ist von der Fauna her anzunehmen, daß hier das Optimum des letzten Interglazials belegt ist. Diese zeitliche Bestimmung ist deswegen so wichtig, weil das Vorkommen der nachgewiesenen Holzarten keineswegs nur auf das Optimum des letzten Interglazials beschränkt ist. Nach der Zusammenstellung der pollenanalytischen Daten durch WELTEN (1981) dürften die meisten dieser Baumarten auch noch in den frühen Interstadialen des letzten Glazials zu erwarten sein. Die Dicke der gefundenen Stämme spricht aber dafür, daß die Bäume nicht im Randbereich ihres Verbreitungsgebietes gewachsen sind.

Die Untergrenze dieser dem letzten Interglazials zugewiesenen Sedimente ist durch weit verbreitete, feinklastische Ablagerungen, dem sogenannten „Oberen Ton“ markiert, der als Leithorizont für die Hydrogeologie gut erfaßt ist. Er wird in keiner der hier betrachteten Kiesgruben durchteuft. Das Alter des „Oberen Tons“ kann aber zur Zeit noch nicht biostratigraphisch beurteilt werden. Bislang wurde er dem Eem-Interglazial, also dem letzten Interglazial zugeordnet (ARBEITSGRUPPE 1980: 19).

Es kann also davon ausgegangen werden, daß die hier beschriebenen Hölzer mit Insekten-Fraßspuren aus dem letzten Interglazial stammen, wobei allerdings nicht sicher auszuschließen ist, daß auch einzelne Hölzer aus den warmen Abschnitten zu Beginn des letzten Glazials kommen. Diese Unsicherheit der stratigraphischen Zuordnung beruht vor allem darauf, daß im gesamten Untersuchungsgebiet der hier interessierende Profilabschnitt unter dem Grundwasserspiegel liegt und das Material nur beim Auskiesen mit Schwimmund Griefbaggern zutage gefördert wird.

Der Befall der Stämme durch die Insekten erfolgte zu Lebzeiten der Bäume oder kurz nach dem Absterben, in jedem Fall aber vor der Einlagerung der Stämme in das Sediment. Inkrustationen und pyritischen Auskleidungen mancher Bohrgänge belegen das fossile Alter der Fraßgänge und Arthropodenreste. Das soll hier extra betont werden, weil RoselT \& FeUsTEL (1960) rezente Nestanlagen von Grabwespen in tertiären Taxodiaceen-Stubben aus der Braunkohle von Frielendorf bei Kassel beschrieben haben. 


\section{Beschreibung der Fraßgänge und Bestimmung der Insekten}

\subsection{Methoden}

Die Holzfragmente wurden je nach Art der erhaltenen Fraßspuren in schmale Scheiben geschnitten, um Verlauf, Größe und Querschnitt der Gänge ermitteln zu können. Ein weiteres Kriterium bildet die Füllung der Gänge z.B. mit Fraßmehl, Nagespänen oder Kot. Die Befunde werden zur Bestimmung mit der Bionomie rezenter Arten verglichen, die in den Tabellen und Befallsbildbeschreibungen von ANONYMUS o.J., BECKER 1949, BRAUNS 1962/66, 1964, Burmeister 1964, Demelt 1966, EsCHerich 1923, Klausnitzer \& SANDER 1978, KOCH 1910, MOURIER \& WINDING 1975, Vité 1952/53 enthalten sind. Die Bestimmung wird allerdings dadurch erschwert, daß diese Bestimmungsliteratur auf vielen Merkmalen aufbaut, die an den fossilen Fraßspuren nicht erfaßbar sind. Andererseits werden einige im fossilen Material durchaus vorhandene Charakteristika nur ungenügend berücksichtigt. Neben der Literatur wurde weiterhin die Fraßsammlung des Hessischen Landesmuseums zum direkten Vergleich genutzt.

\subsection{Befunde an Hölzern von Picea/Larix}

RSH 20 - Fraßgänge der Gemeinen Holzwespe, Paururus juvencus ( $L$ ) (Siricidae, Hymenoptera) s. Abb. 2 und 10.

RSH 21 - Zahlreiche hakenförmige Puppenwiegen von Fichten- oder Lärchenböcken, Tetropium sp. (Cerambycidae, Coleoptera) s. Abb. 3. 2/3 der Puppenwiegen sind von Schwarzoder von Buntspechtarten (Dryocopus martius bzw. Dendrocopus sp., Picidae, Aves) aufgeschlagen. Verlassene Bockkäfergänge wurden sekundär von weiblichen Grabwespen der Gattungen Trypoxylon/Pison (Sphecidae/Hymenoptera) zum Brutkammerbau genutzt. Mehrere Prosomata eingetragener Kreuzspinnen, Gattung Araneus (Araneidae, Arachnida), wurden in den Gängen sichergestellt s. Abb. 4.

RSH 28 - Zahlreiche Wohn- und Fraßgänge eines Bohrrüsselkäfers (Curculíonidae, Coleoptera). Sie waren von Braconiden, Bethyliden oder Chalicididen (Hymenoptera) parasitiert. Einzelne Larvenfraßgänge eines Scheibenbockes, Callidium sp. (Cerambycidae, Coleoptera).

RSH 20: Das Holzstück enthält mehrere Gangsysteme. Sie sind im Querschnitt kreisrund und fest mit Bohrmehl gefüllt. Die verschiedenen Gangsysteme nehmen einen ähnlichen und daher wohl nicht zufälligen Verlauf. Sie beginnen in der äußersten erhaltenen Schicht und haben nur einen Durchmesser von etwa $1 \mathrm{~mm}$. Sie durchziehen zunächst das Frühholz, gehen dann aber radiär durch mehrere Schichten in die Tiefe des Stammes, um dann wieder schichtenparallel durch das Frühholz zu verlaufen. Dabei nimmt ihr Durchmesser beständig zu. Schließlich führt der Gang wieder radiär zur Holzoberfläche zurück, wo er unweit seines Beginns mit kreisrunder Offnung von etwa $6 \mathrm{~mm}$ endet. Die Abb. 10 zeigt den typischen Verlauf eines dieser Gänge, bei dem zusätzlich vier blind endende Seitengänge auffallen.

Der schlingenförmige Verlauf der Gänge durch das Holz ist typisch für Holzwespen (Siricidae/Hymenoptera). Auch das sehr fest verbackene Bohrmehl sowie der kreisrunde Querschnitt der Gänge sind typisch für diese Insekten. Die kleine Familie der Holzwespen umfaßt nur fünf artenarme Gattungen, von denen drei (Sirex, Paururus, Xerris) an Conifera und zwar unterschiedlos an alle einheimischen Conifera-Arten gehen. Als einzige Art dieser 3 Gattungen wenden sich die Larven von Paururus juvencus um, nachdem sie sich ein Stück durch das Holz genagt haben und fressen sich durch ihr eigenes Bohrmehl wieder zurück. Auf diese Weise verursacht diese Art blind endende Gänge. 


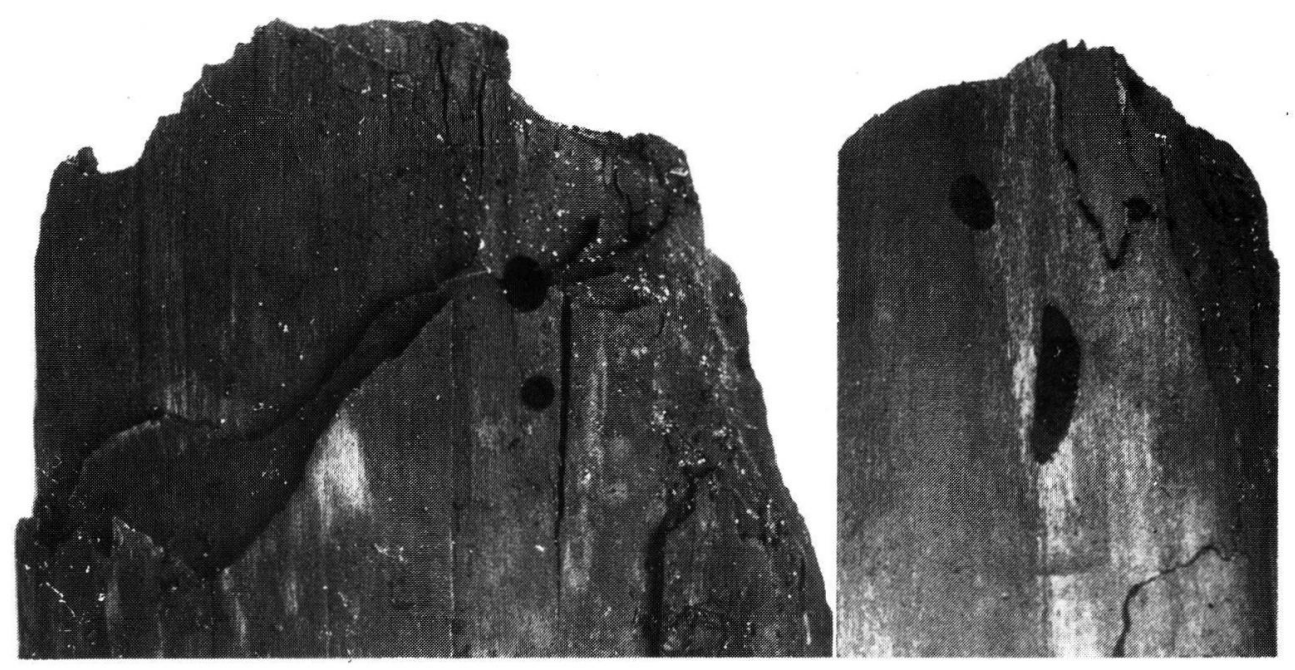

Abb. 2: Holz und Picea/Larix mit Fraßgängen der Gemeinen Holzwespe, Paururus juvencus. Die Gänge sind mit Fraßmehl fest verstopft (siehe auch Abb. 10) - (RSH 20, Bibilis, letztes Interglazial).

Die beiden anderen Arten fressen sich in einem einfachen Schlingengang durch das Holz. Da das vorliegende Stück Kiefernholz mehrere dieser Blindgänge aufweist, muß es sich um die Art Paururus juvencus handeln. Dafür spricht auch, daß ein relativ dünner Stamm befallen wurde, der von einer entsprechend dünneren Borkenschicht bedeckt war. Die Stärke der Borkenschicht spielt nämlich bei der Eiablage der verschiedenen Holzwespenarten eine wesentliche Rolle. Nur die Arten mit langem Legebohrer (z.B. Sirex gigas) legen ihre Eier in den bodennahen Bereich starker Stämme, während die Holzwespen mit kürzerem Legebohrer, und dazu gehört Paururus juvencus, die oberen, dünneren Stammpartien bevorzugen.

RSH 21: Mehrere hochovale Löcher von $4 \mathrm{~mm} \times$ 2,4 $\mathrm{mm}$ Durchmesser, an die sich ein Hakengang von meist $6 \mathrm{~cm}$ Länge anschließt (Abb. 3). Bei den Hakengängen handelt es sich um die Puppenwiegen eines Bockkäfers (Cerambycidae). Die Größe der Puppenwiege, die sehr scharfe Wendung des Ganges und die senkrechte Stellung der ovalen Öffnung sind typisch für die Vertreter der Gattung Tetropium. Diese Gattung umfaßt drei Arten in Mitteleuropa: T. castaneum (L), T. fuscum (F.) und T. gabrieli, WEISE. Der Lärchenbock, T. gabrieli, befällt ausschließlich Lärchen (Larix sp.), während die beiden anderen Arten fast nur an Fichten (Picea sp.) auftreten. T. castaneum befällt außerdem besonders im $\mathrm{E}$ und $\mathrm{N}$ seines Verbreitungsgebietes ebenso regelmäßig Kiefer (Pinus sp.). Da die Fraßbilder, speziell die Puppenwiegen, keine Unterscheidung der 3 Arten gestatten und auch holzanatomisch die Hölzer von Picea und Larix nicht unterschieden wurden, ist eine über das Gattungsniveau hinausgehende Bestimmung des Käfers hier nicht möglich.

Am vorliegenden Holz ist weiterhin deutlich die Arbeit von Spechten überliefert (Abb. 3). Vor allem der Schwarzspecht (Dryocopus martius) und die Buntspechtarten (Dendrocopus sp.) gehören zu den regelmäßigen Begleitern eines Befalls mit Fichtenböcken und weisen oft auf einen Befall hin, bevor dieser direkt durch die Fraßwirkung bemerkt wird (EsCHERICH 1923). Die von $\operatorname{dem}(n)$ Specht(en) gemeißelten Schlagtrichter weisen eine 

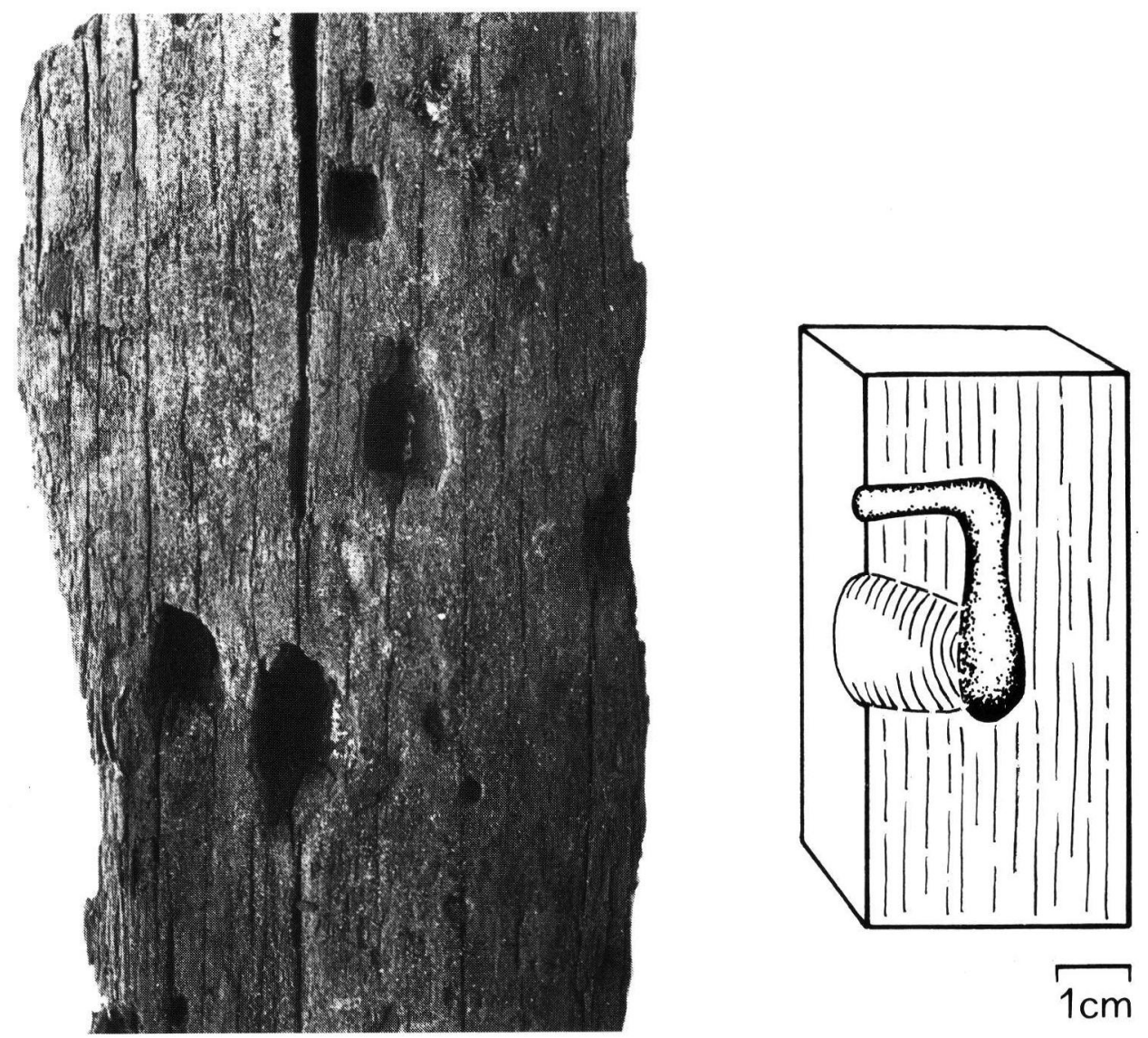

Abb. 3: Holz von Picea/Larix mit hakenförmigen Puppenwiegen von Fichten- oder Lärchenböcken, Tetropium sp., mit hochovalen Fluglöchern von maximal $5 \mathrm{~mm}$ Durchmesser. Zwei Drittel der Puppenwiegen in diesem Holz sind von Schwarz- oder Buntspechten aufgeschlagen (trichterförmige, unregelmäßige Gruben) - (RSH 21, Biblis, letztes Interglazial).

flachere und eine steilere Wand auf, die aus ihrer Orientierung zum einen anzeigen, daß der Käferbefall am stehenden und nicht etwa an einem bereits umgestürzten Baum erfolgte und zum anderen beim gefundenen Holzstück die Festlegung von „oben“ und ,unten“ ermöglichen. Die Anwendung dieser zusätzlichen Erkenntnisse auf den Käferbefall ergibt, daß die Hakengänge senkrecht nach unten führen, was mit den Beobachtungen rezenten Befalls dieser Bockkäfer übereinstimmt. Die Schlagtrichter führen zu den Bockkäferpuppenwiegen (Abb. 3) und lassen die Annahme zu, daß etwa 2/3 der verpuppungsreifen oder bereits verpuppten Käferlarven dem(n) Specht(en) zum Opfer gefallen sind.

In einem der Gänge wurden außerdem Kutikulateile gefunden, die sich als Prosomata mehrerer Spinnen erwiesen. Darunter ist eine Spinne (Abb. 4), deren Körper in der Exuvie noch fest eingeschlossen war, sich also gerade in der Häutungsphase befunden hat. Die dichte, regelmäßige Anordnung der Spinnenteile spricht gegen ein Winterlager. Die Spinnen sind vielmehr durch eine Wespe eingetragen worden und sollten deren Nachkommen als Nahrung dienen. Da die Spinnen jedoch noch beinahe unversehrt geblieben sind, nur 


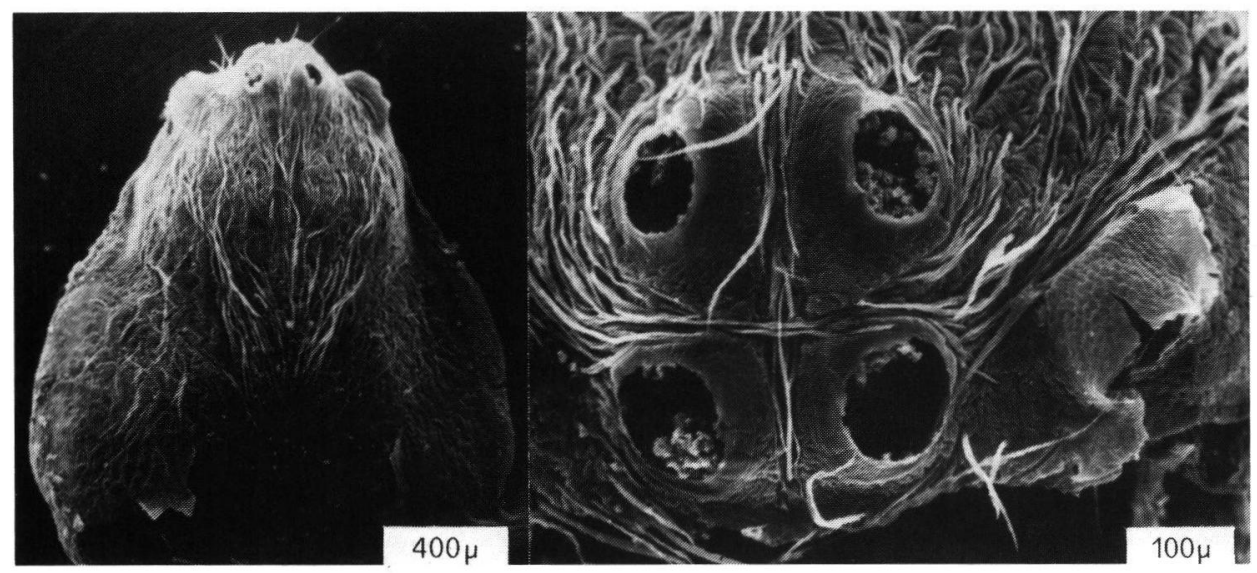

Abb. 4: Prosoma einer der Spinnen der Gattung Araneus sp. (links: Dorsalansicht, rechts: Augenregion bei stärkerer Vergrößerung), die in den Gängen der Bockkäfer gefunden wurden. Wahrscheinlich hatte ein Weibchen von Trypoxylon (Grabwespe) in dem offenen Gang mit der Anlage ihres Nestes begonnen. Die abgebildete Spinne, eine von mehreren, befand sich gerade in der Häutungsphase, als sie von der Grabwespe durch einen Stich paralysiert wurde. Die Exuvie wurde vor der Aufnahme abgehoben, wodurch sich die hervorragende Erhaltung des behaarten Prosoma erklärt (siehe auch Abb. 3) - (RSH 21, Biblis, letztes Interglazial).

das weichhäutigere Opistosoma fehlt, muß der Nestbau - evtl. durch den Tod des Wespenweibchens - unterbrochen worden sein. Wegwespen (Pompilidae) und Grabwespen (Sphecidae) sind als Spinnenjäger bekannt. Die Pompilidae tragen jedoch immer nur eine Spinne pro Nest ein und nisten überdies im Boden. Die Spinnenjäger innerhalb der Sphecidae werden im wesentlichen den vier Gattungen Pison, Miscophus, Sceliphron und Trypoxylon zugerechnet (BISCHOFF 1957, ESCHERICH 1942, FRIESE 1926, JACOBS \& RENNER 1974, schriftl. Mitt. Dr. Bachmaier, München 1984 und mdl. Mittl. M. Sorg, Neunkirchen 1984). Doch nur die wenigen Arten der Gattungen Trypoxylon und Pison nutzen die verlassenen Gänge von holzbohrenden Insketen für ihren Nestbau, in den sie mehr als 30 durch einen Stich gelähmte Spinnen eintragen. Vermutlich war ein Weibchen einer dieser beiden Gattungen am vorliegenden Holzstück mit dem Nestbau beschäftigt.

RSH 28: Das vermutlich durch Rotfäule zersetzte Fichtenholz dürfte am Boden gelegen haben und war vielleicht teilweise mit Laub bedeckt. Das ergibt sich aus dem Pilzmyzel (Abb. 5) sowie aus einer Wurzel der Waldrebe (Clematis vitalba), die das Holz durchwachsen haben. Von der ehemaligen Außenseite des Stammes her ist das Holz mehrere Zentimeter tief mit runden Gängen durchsetzt, die z.T. mit Sediment aber auch mit Kotpillen locker gefüllt sind. Die Gänge, deren Durchmesser etwa $3 \mathrm{~mm}$ beträgt, nehmen einen unregelmäßigen Verlauf und durchkreuzen sich sogar. Die in den Gängen enthaltenen Kotpillen (Abb. 6) lassen einen Befall durch Bohrrüsselkäfer (Cossoninae, Circulionidae) erkennen, die feuchtes, brüchiges Holz bevorzugt besiedeln. Das Fraßbild alleine ist nicht diagnostisch, denn es ähnelt dem der Klopfkäfer (Anobiidae, Coleoptera), wie sie in RSH 25 belegt sind. Von den 30 Arten aus der Unterfamilie der Bohrrüsselkäfer wurden 7 Arten, die zu 5 Gattungen gehören, in Fichtenholz und anderen Nadelhölzern festgestellt. Daher ist eine genauere Bestimmung vorerst nicht möglich. 


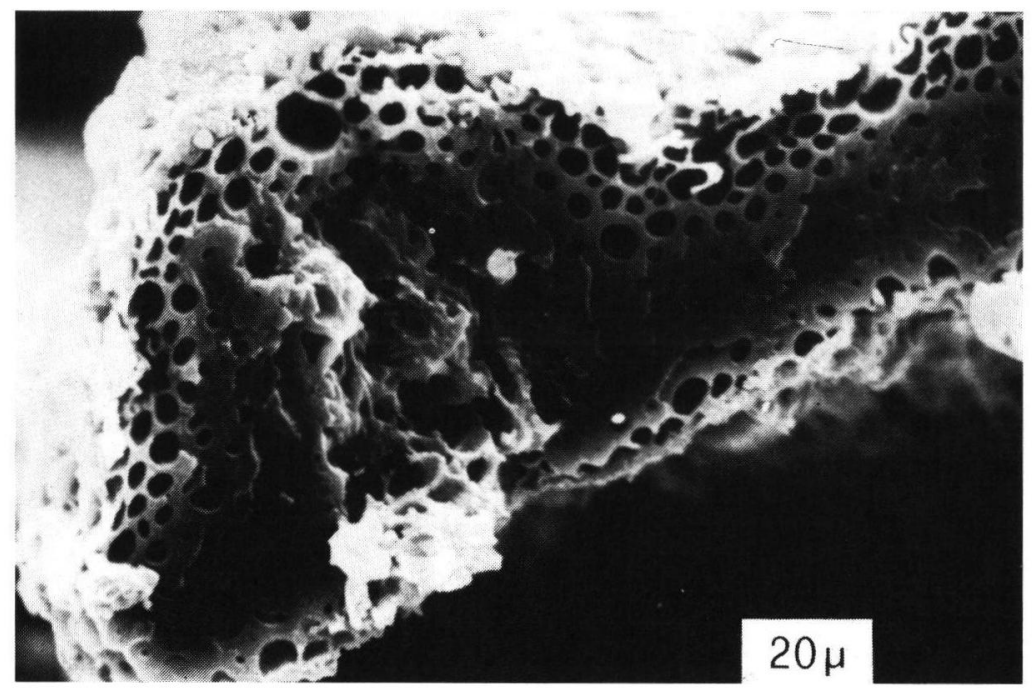

Abb. 5: Pilzmyzel im Holz von Picea/Larix verrät, daß dieses Holz längere Zeit feucht am Boden gelegen hat, ehe es eingebettet wurde. Abgebildet ist ein Ausschnitt aus der Bruchkante des Pilzmyzels mit großlumigen Hyphen an der Außenseite - (RSH 28, Groß-Rohrheim, letztes Interglazial).

Daneben fallen eine sehr große Anzahl winziger Gänge auf, die zwischen 0,15 mm und $0,25 \mathrm{~mm}$ Größe variieren. Diese Gänge lassen sich von ihrer Mündung in der Holzoberfläche bis zu den Rüsselkäfergängen zurückverfolgen. Sie enthalten weder Kot noch Bohrmehl und ändern ihre Größe nicht. Es bieten sich zwei Interpretationen an. Entweder handelt es sich um die Gänge der Eilarven der Rüsselkäfer, oder es sind die Ausgänge von parasitischen Wespen (Braconidae, Bethylidae, Chalcididae/Hymenoptera). Für letztere spricht, daß sie in ihrer Größe konstant bleiben, daß sie keine Kotpillen enthalten und daß vor allem die Zwischengrößen zwischen diesen nur 1/5 mm messenden Gängen und den $3 \mathrm{~mm}$ starken Rüsselkäfergängen nicht auffindbar sind. Die beachtliche Anzahl dieser auffällig kleinen Ausgänge und ihre recht große Variationsbreite sind bemerkenswert. Der geringe Durchmesser der Ausgänge schließt z.B. einen Erzwespenbefall nicht aus, sind doch gerade unter ihnen die kleinsten bekannten Insekten vertreten.

Das Holzstück wird außerdem von einigen sehr flachovalen Fraßgängen durchzogen, die mit Bohrmehl fest verstopft sind. Ihre Größe variiert von $2,5 \mathrm{~mm}$ x $7 \mathrm{~mm}$ bis $3,5 \mathrm{~mm}$ x $8,5 \mathrm{~mm}$. Es handelt sich hierbei um die Fraßgänge von Bockkäferlarven (Cerambycidae/Coleoptera). Der mit 2,4:1 bzw. 2,8:1 recht flache Gang spricht gegen einen Befall mit Tetropium-Arten, die an einem anderen Holzfragment (RSH 21) aufgrund ihrer sehr charakteristischen hakenförmigen Puppenwiegen festgestellt wurden. Das den Gängen entnommene Bohrmehl enthält nur schuppenförmige Nagespäne, was die in allen Nadelhölzern verbreiteten Scheibenböcke (Callidium sp.) auszeichnet (BECKER 1950). 


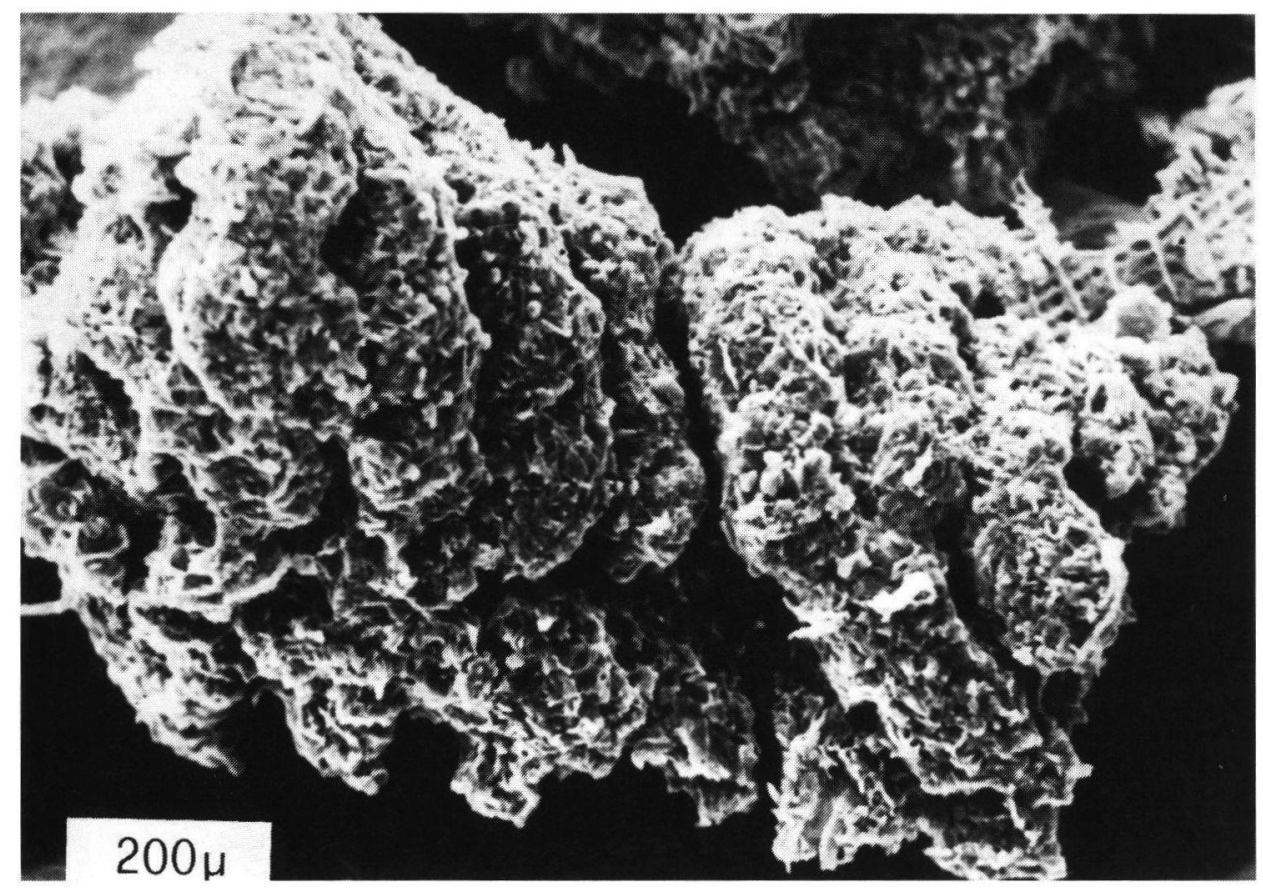

Abb. 6: Unregelmäßig geformte Kotpillen mit Holzsplittern eines Bohrrüsselkäfers (Cossoninae, gen. sp.) aus dem Holz von Picea/Larix - (RSH 28, Groß-Rohrheim, letztes Interglazial).

\subsection{Befunde an Hölzern von Abies alba}

RSH 23 - Mehrere Leitergangsysteme vom Nutzholzborkenkäfer Xyloterus lineatus OLIv. (Scolytidae/Coleoptera) (Abb. 7).

RSH 24 - Das Holz wird von zahlreichen Gängen durchzogen, in denen drei Larven des Holzinsektes sichergestellt werden konnten, die als Buprestis sp. (Buprestidae, Coleoptera) (Abb. 8) bestimmt wurden. Das Holzstück enthält weiterhin zwei viertelkreisförmige Puppenwiegen, die ebenfalls von diesen Käfern stammen können (Abb. 11).

RSH 23: Mehrere kreisrunde Gänge mit auffallend schwarzer Auskleidung ziehen radiär in das Innere des Astes. Dort zweigen waagerecht angelegte Seitengänge ab, die ihrerseits bis zu 15 Paar kurze, senkrecht verlaufende Blindgänge in sehr regelmäßiger Anordnung aufweisen (Abb. 7 und 12). Das Gangsystem wurde von Xyloterus lineatus (Scolytidae/Coleoptera) angelegt. Die weiblichen Käfer stellen zunächst den in das Holz führenden Radiärgang her, von dessen Ende sie meist zwei konzentrische Brutarme abzweigen lassen. Diese erhalten oben und unten in regelmäßigem Abstand zueinander Eikammern, in die jeweils ein Ei gelegt wird. Die aus den Eiern schlüpfenden Larven erweitern die Eikammern zu einem kurzen, zylinderförmigen Gang. So entsteht das Leitergangsystem. Die Nahrung der Käfer und Larven besteht aus den Nährzellen, die ihnen in den Gängen kultivierte Ambrosia-Pilze liefern. Käfer und Larven dieses Borkenkäfers sind mycetophag. Sie reihen sich in die pilzüchtenden Käfer ein, die Sporen ,ihrer“ Ambrosia-Pilze in speziellen Taschen 


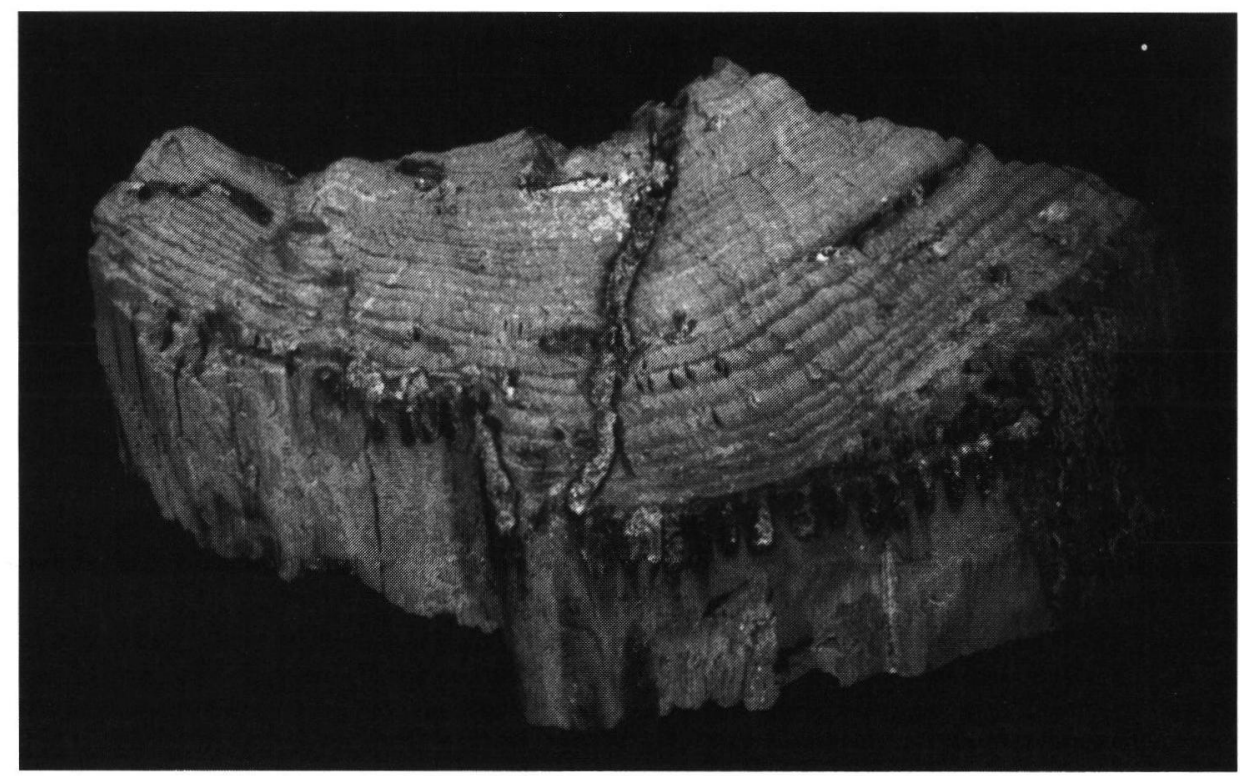

Abb. 7: Holz von Abies alba mit dem Ausschnitt aus dem Leitergangsystem von Xyloterus lineatus, dem Nutzholzborkenkäfer. Angeschnitten sind die in Jahresringen angelegten Brutarme mit den kurzen Larvenfraßkammern. Diese Insekten ernähren sich von Abrosia-Pilzen, deren Reste den Belag der Gänge bilden (siehe auch Abb. 12) - (RSH 23, Groß-Rohrheim, letztes Interglazial).

(Mycetangien) in der Mandibel mit sich führen und bei der Besiedelung in den frisch angelegten Gängen ,,aussäen“. In vom Käfer verlassenen Gängen stirbt der Pilz unter Schwarzfärbung ab. Dies erklärt die auffallende Auskleidung der vorgefundenen Leitergänge.

RSH 24: Das Holz weist mehrere in erster Linie faserparallel verlaufende Larvenfraßgänge auf, die sehr unregelmäßig durch das Holz ziehen und oftmals etwas blasig ausgefressen sind. Die bis maximal 3 x 7,5 mm dicken Fraßgänge sind mit sehr feinem Fraßmehl so fest verstopft, daß es oftmals schwierig ist, den Verlauf der Gänge durch das fossile Holz zu verfolgen. In den Gängen wurden drei Larven gefunden, die u.a. aufgrund der gezähnten Mandibel (Abb. 8) von Herrn Dr. h.c. S. CYMOREK, Krefeld, als Buprestis sp. (Buprestidae, Coleoptera) bestimmt wurden. Ohne direkte Verbindung, aber wohl zum gleichen Befall gehörend, enthält das Holz weitere faserparallel angeordnete, ovale Gänge. Sie sind ebenfalls mit sehr feinem Bohrmehl fest verstopft, münden dann aber, ohne die Richtung oder den Querschnitt zu ändern, in Puppenwiegen, die kein Bohrmehl mehr enthalten. Die beiden beobachteten Puppenwiegen, die zunächst weitere $3 \mathrm{~cm}$ faserparallel verlaufen, ziehen dann in weitem Bogen waagerecht nach außen. Da die jetzige Mündung der Gänge seitlich in der Bruchfläche des Holzes liegt, müßten die Puppenwiegen nach dem Verlauf der Jahresringe zu urteilen mindestens 12 bzw. $13 \mathrm{~cm}$ tief im Inneren des Holzes gelegen haben. Bemerkenswert ist noch die Orientierung des querovalen Ganges. Sein größter Durchmesser liegt im vertikalen Abschnitt in beiden Fällen parallel zum Radius des Holzes, dreht sich dann im Bogen so, daß er im radialen Abschnitt schräg zur Längsachse des Holzes liegt (Abb. 11). Da dies bei beiden Puppenwiegen der Fall ist, dürfte es nicht zufällig sein. Die Puppenwiegen könnten ebenfalls von Buprestis sp. angelegt sein. 


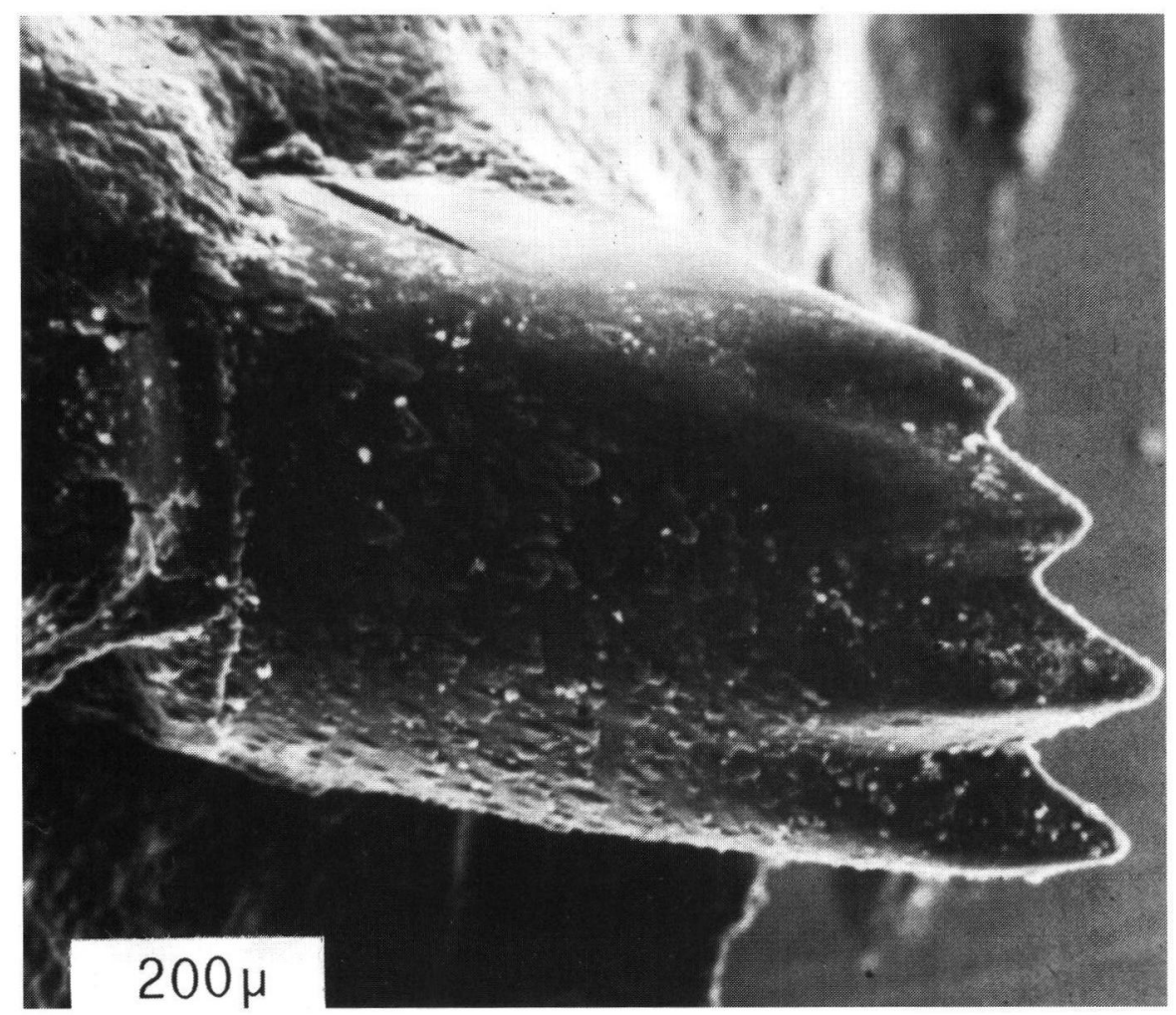

Abb. 8: Mandibel einer Larve von Buprestis sp. aus einem larvalen Fraßgang im Holz von Abies alba - (RSH 24, Groß-Rohrheim, letztes Interglazial).

\subsection{Befunde an Hölzern von Ulmus sp.}

RSH 22 - Ein quer zur Faserrichtung des Holzes verlaufender Fraßgang eines unbestimmten Bockkäfers (Cerambycidae, Coleoptera, gen. sp.). Puppenwiege und Flugloch einer unbekannten Insektenart (?Anobiidae, Coleoptera). (Abb. 13).

RSH 26 - Bogenförmige Puppenwiegen einer nicht bekannten Insektenart.

RSH 22: Das Holzstück wird von einem hochovalen Gang durchzogen, der auf einer Länge von $6.5 \mathrm{~cm}$ erhalten ist. Die Mündung des Ganges liegt parallel zur Längsachse des Holzes und hat die Maße 4 x 8,5 mm. Der Verlauf des Ganges ist unregelmäßig. Er enthielt kein Bohrmehl. Die Bestimmung ist wegen der geringen Anzahl verwertbarer Merkmale sehr problematisch. Das Alter der Larve, die diesen Gang genagt hat, ist nicht zu erschließen und damit bietet der Durchmesser des Ganges nur wenig Anhaltspunkte. Ebenso ist die Tatsache, daß der Gang kein Bohrmehl enthält, nicht überzubewerten, da dies herausgewaschen worden sein könnte.

Das Holz enthält weiterhin einen kreisrunden Gang von etwa 0,9 mm Durchmesser, der radial zu einer Erweiterung führt, die 1,2 cm unter der überlieferten Holzoberfläche angelegt ist. Diese Erweiterung liegt faserparallel. Von dort führt der Gang weiter radial durch das Holz, das er an der gegenüberliegenden Bruchfläche verläßt. Die Gänge enthielten kein 
Bohrmehl. Bei der Erweiterung handelt es sich um eine Puppenwiege. Die Larve muß im Inneren des Holzes herangewachsen sein, ihre Fraßgänge sind jedoch nicht erhalten. Ptilinus-Arten legen Gänge dieses Durchmessers an und verpuppen sich in einer Puppenwiege, die der im vorliegenden Holzstück gefundenen entspricht. Möglicherweise ist daher Ptilinus sp. (Anobiidae, Coleoptera) der Verursacher des Ganges (Abb. 13).

RSH 26: Mehrere runde Gänge mit einem Durchmesser von 2,4 mm bis 3,9 $\mathrm{mm}$ ziehen in einem halbkreisförmigen Bogen durch das Holz und enden dann unmittelbar unter der Holzoberfläche (Abb. 14). Manche der Bogengänge, die anscheinend weniger tief in das Holz geführt wurden, sind aufgeschnitten, so daß von ihnen nur eine längliche Vertiefung in der Oberfläche des Holzfundes verblieben ist. Es handelt sich um die Puppenwiegen einer Käferart, deren Larven sich direkt unter der Rinde ernähren und nur zur Verpuppung tiefer in den Splint eindringen. Eine Determination steht noch aus.

\subsection{Befunde an Hölzern von Fraxinus excelsior}

RSH 25 - Fraß- und Wohngänge des Klopfkäfers Anobium sp. (Anobiidae, Coleoptera).

Das Aststück weist zahlreiche im Querschnitt runde Gänge auf, die das Splintholz in unregelmäßigem Verlauf durchziehen. Der Durchmesser der Gänge beträgt etwa 2,5 mm.

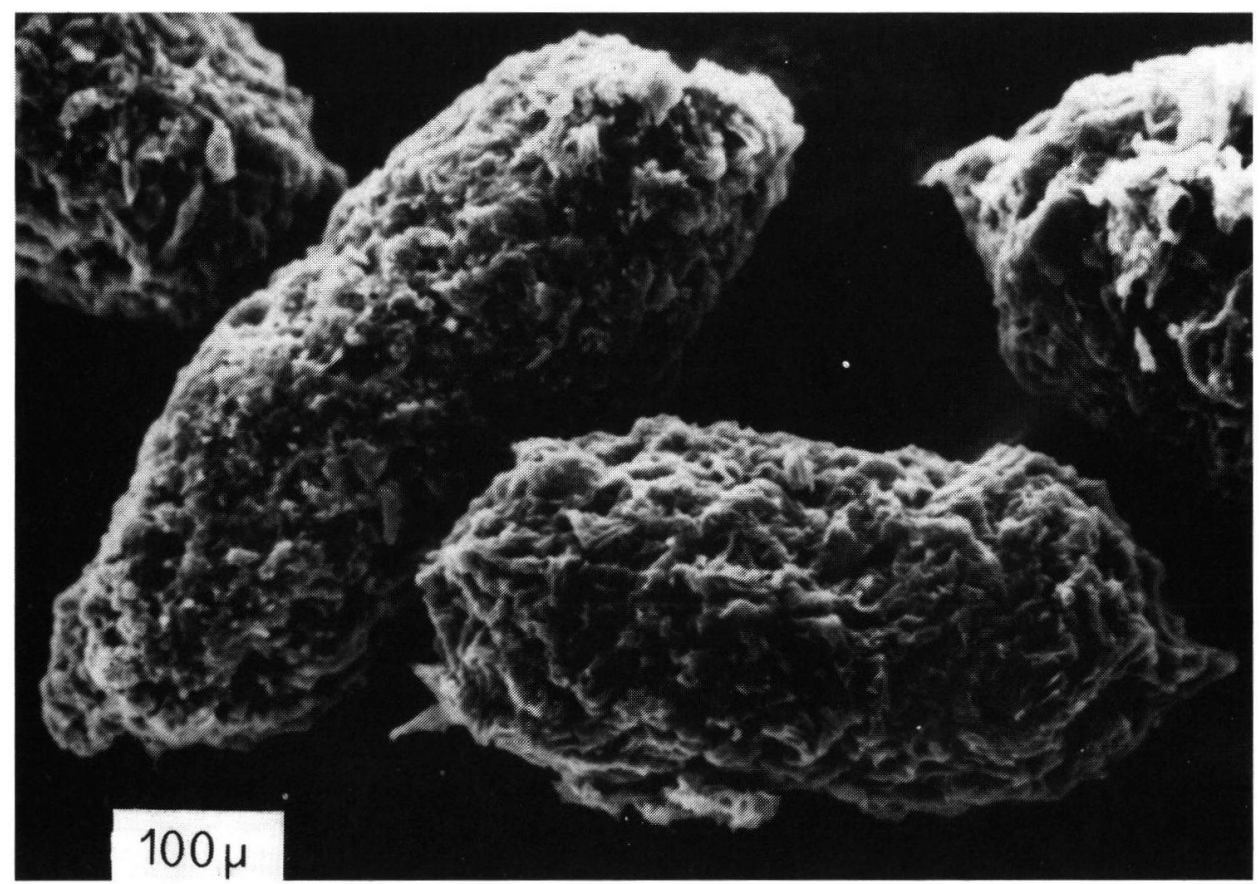

Abb. 9: Kotpillen aus den Fraßgängen von Anobiidenlarven in Eschenholz. Die längliche, an einer Seite zugespitzte Form der Kotpillen ist charakteristisch für Käfer der Gattung Anobium - (RSH 25, Geinsheim, letztes Interglazial). 
Viele Gänge sind mit länglichen Kotpillen (0,7 x 0,2 mm) gefüllt (Abb. 9). Es handelt sich um die Fraßgänge eines Klopfkäfers der Gattung Anobium. Die Form der Kotbällchen ist für Arten dieser Gattung sehr charakteristisch.

Eine Art dieser Gattung, Anobium punctatum DEG., ist in der jungpleistozänen Fauna von Tattershall, Lincolnshire, England, nachgewiesen (GIRLING 1974, zit. in COOPE 1979). A. punctatum ist heute vorwiegend synanthrop in Nadel- wie auch Laubhölzern verbreitet und meidet bei Harthölzern wie Buche, Eiche und Esche das Kernholz. Seine Gänge haben einen Durchmesser bis maximal 3,3 mm, so daß es sich bei den hier vorliegenden Gängen mit ihrem etwas geringeren Durchmesser um die Fraßspuren einer anderen Art handeln müßte, zumal die Kotpillen in Form und Größe auch nicht mit denen von A. punctatum übereinstimmen.

\section{Das biologische Umfeld der Holzinsekten}

Nur eine der aufgesammelten Holzproben (RSH 24) hat in den Fraßgängen Käferlarven und damit den Erzeuger der Gänge überliefert. Mehrfach wurden Bohrmehl, Fraßspäne und Kot gefunden, die zum Teil für einzelne Gattungen charakteristisch sind. Im wesentlichen muß sich allerdings die Bestimmung der Holzinsekten auf den Verlauf und die Form der Gänge sowie die Art ihrer Verfüllung stützen. In Einzelfällen kann auch die Bestimmung der Holzart zur Identifizierung der Holzinsekten beitragen, die unter Hinzuziehung aller genannten Indizien weitgehend bis ins Gattungsniveau, gelegentlich bis zum Artniveau erfolgen konnte.

Vergleichbare Arbeiten über fossile Holzinsekten liegen unseres Wissens noch nicht vor, so daß die Bestimmung der Fraßspuren in den jungpleistozänen Hölzern vorerst nur als Vergleich mit der Bionomie rezenter Arten erfolgt. Der Fehler dürfte hierbei aber gering sein, da z.B. CoOPE $(1969,1974,1975,1977,1979)$ an Hand von Käferfaunen aus dem englischen Pleistozän zeigen konnte, daß etwa $99 \%$ der pleistozänen Käfer der rezenten Fauna angehören. Aus dem englischen Pleistozän konnte er unter 700 Käferarten nur 5 finden, die in der rezenten Fauna nicht mehr vertreten sind.

In Tabelle 1 werden die hier an fossilen Hölzern nachgewiesenen Arthropoden aufgeführt und nach der forstbiologischen Gliederung als primäre, sekundäre oder tertiäre Holzinsekten klassifiziert. Weiter ist angegeben, ob sie durch eigene Enzyme befähigt sind, Holz zu verdauen oder sich der Hilfe symbiontischer Pilze bedienen. Neben den Holzinsekten ist auch deren Räuber, ein Buntspecht (Dryocopus sp.) oder Schwarzspecht (Dendrocopus sp.) durch seine Hackspuren eindeutig belegt. Die Liste stellt dennoch nur einen kleinen Ausschnitt aus der viele hundert Arten umfassenden Gruppe der holzbewohnenden Insekten dar. Dennoch sind interessanterweise nahezu alle biologischen Typen vertreten. Es zeichnet sich damit, wenn auch nur andeutungsweise, die Lebensgemeinschaft xylobiontischer Insekten $a b$, in der jede Art mehr oder minder unmittelbar von dem Vorhandensein einer anderen Art abhängig ist (WEBER \& WEIDNER 1974).

Die Mehrzahl der Holzinsekten ist entweder auf Nadel- oder auf Laubholz angewiesen. Paururus juvencus (RSH 20), Tetropium sp. (RSH 21) sind typische Nadelholzinsekten. Manche sind monophag und an eine bestimmte Baumart gebunden, wie Tetropium gabrieli, der Lärchenbock, der nur an Lärchen vorkommen kann und möglicherweise die Puppenwiegen des Holzes RSH 21 angelegt hat. Gründe für die Spezialisierung auf bestimmte Holzarten sind nur ungenügend bekannt. Die Entscheidung, welcher Baum oder welches Holz befallen wird, trifft das eierlegende Weibchen, das sowohl die Holzart wie den Zustand des Holzes vor der Eiablage sehr genau auf seine Eignung prüft. Damit wird deutlich, daß neben 


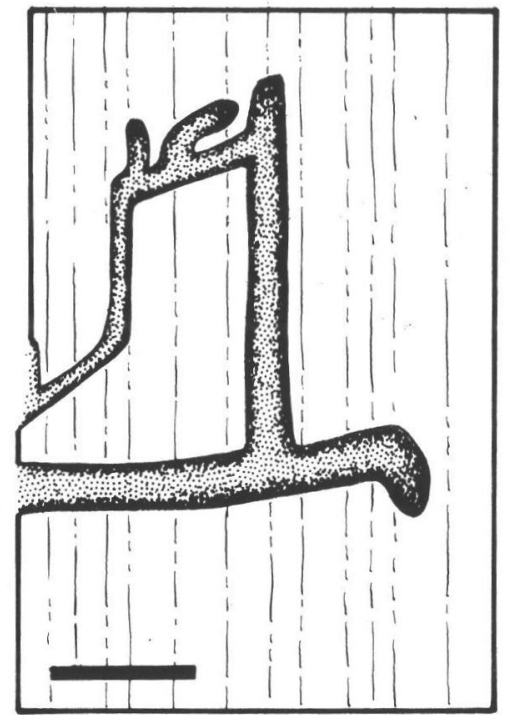

10

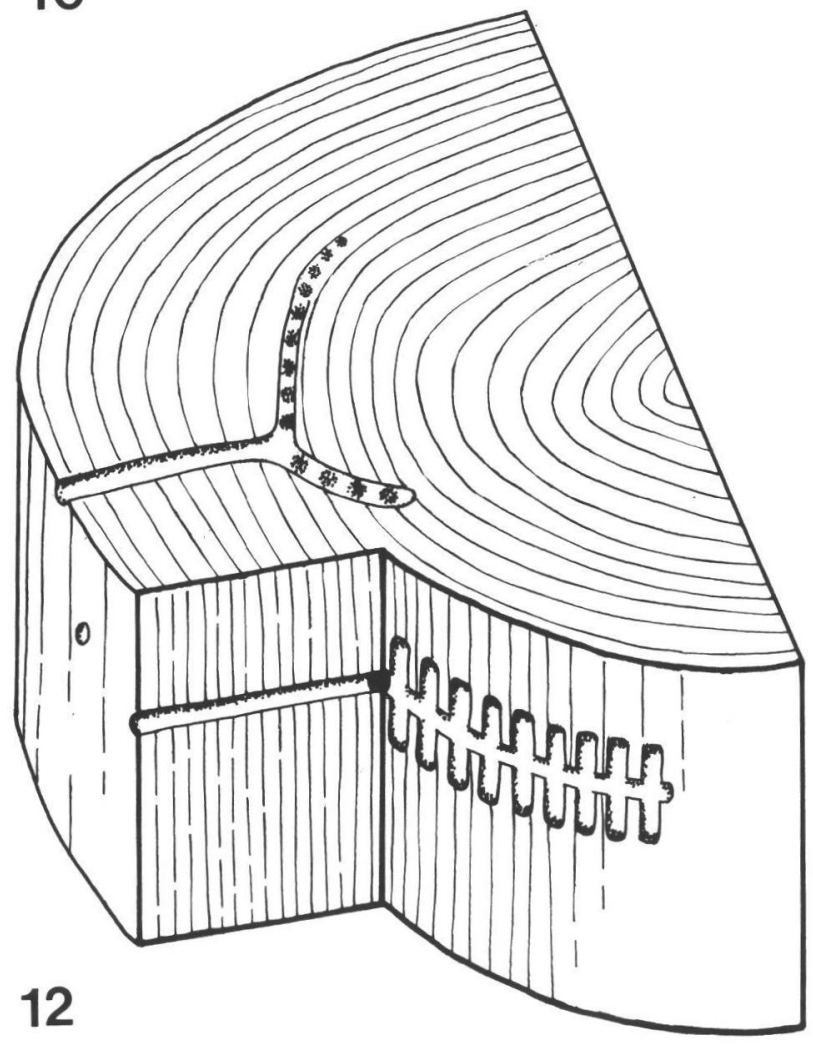

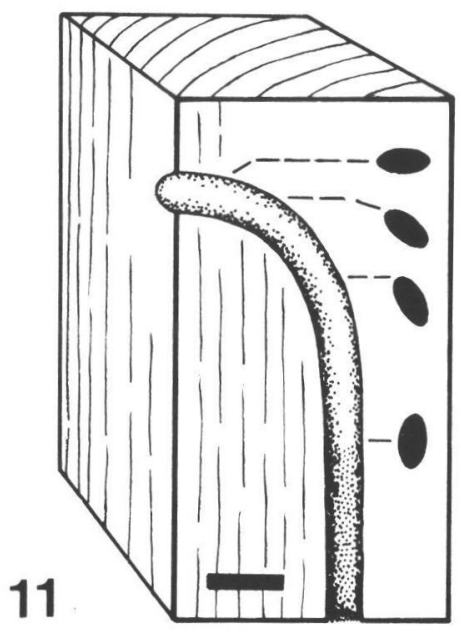
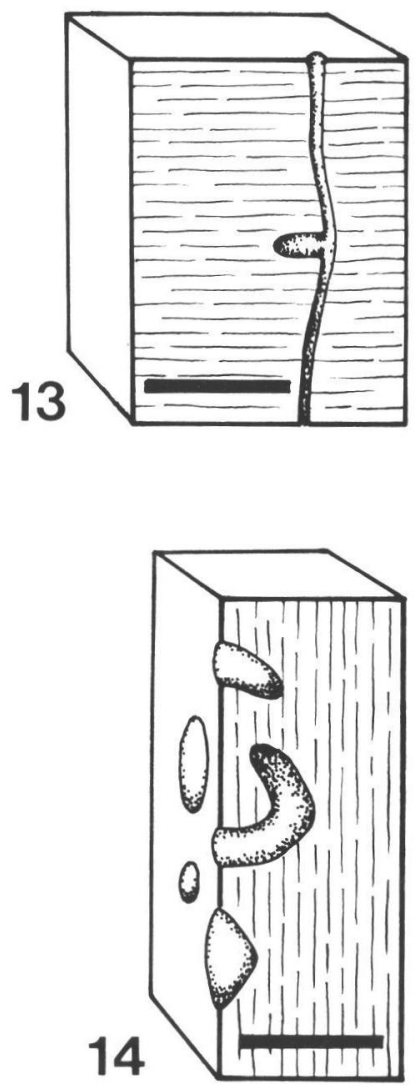
Abb. 10-14: Halbschematische Darstellung der Fraßspuren von Holzinsekten in Hölzern des letzten Interglazials aus der nördlichen Oberrheinebene. Maßstäbe jeweils $1 \mathrm{~cm}$.

Abb. 10: Ein Schlingengang der Gemeinen Holzwespe, Paururus juvencus, in eine Ebene projeziert. Die Larve hat sich mehrmals umgewendet und durch ihren mit Kot und Fraßmehl gefüllten Gang zurückgefressen. Dadurch sind die für diese Art typischen Blindgänge entstanden (RSH 20, Biblis).

Abb. 11: Bogenförmige Puppenwiege von Buprestis sp. im Holz von Abies alba, die sich unmittelbar an den vertikal verlaufenden Larvenfraßgang anschließt. Auffällig ist der in sich verwundene ovale Querschnitt (RSH 27, Groß-Rohrheim).

Abb. 12: Modell des Brutganges von Xyloterus lineatus, der aus einem Radiärgang und meistens zwei konzentrischen Brutarmen besteht, von denen die senkrecht stehenden Larvenkammern abzweigen, wie sie in RSH 23 aus Groß-Rohrheim fossil belegt sind (siehe auch Foto Abb. 7) (nach NÜsSLIN \& RÜMBLER.

Abb. 13: Ein quer zur Faserrichtung verlaufende Gang mit waagerechter Puppenwiege, vielleicht von (?) Ptilinus sp. (RSH 22, Groß-Rohrheim).

Abb. 14: Halbkreisförmige Bogengänge einer noch unbestimmten Insektenart. Manche Bogengänge sind nur teilweise überliefert, weil Rinde und Teile des Bastes fehlen, so daß hier nur längliche Vertiefungen in der Holzoberfläche erhalten sind (RSH 26, Groß-Rohrheim).

der Holzart auch der Zustand des Holzes von entscheidender Bedeutung ist. Dieser bestimmt die forstbiologische Gliederung in primäre, sekundäre und tertiäre Holzinsekten.

Die primären Insekten bilden die kleinste Gruppe. Sie sind in der Lage, das Holz eines lebenden Baumes zu besiedeln und leiten damit seinen Abbau ein. Es genügt eine geringfügige Schwächung des Baumes durch übermäßigen Raupenblattfraß, durch eine längere Trockenperiode oder durch eine Grundwasserabsenkung, um den in RSH 21 nachgewiesenen Splintböcken die erfolgreiche Besiedlung zu ermöglichen. Durch ihre Fraßtätigkeit im Splint wird der Saftstrom des Baumes unterbrochen, so daß der nun stark geschwächte Baum von den sekundären Holzinsekten angegriffen werden kann. Zu diesen zählen Holzwespen (RSH 20), einige Scheibenbockarten (RSH 28) und die Nutzholzborkenkäfer (RSH 23). Mit dem Absterben des Baumes wird das Holz zunehmend von Bakterien und Pilzen angegriffen und bietet nun auch den Prachtkäfern (RSH 24) Lebensmöglichkeiten. In dem zuletzt mulmigen, faulenden Holz finden die tertiären Insekten ihren Lebensraum, die diesen Prozeß weiter beschleunigen. Es sind u.a. Scheibenböcke (RSH 28) und Bohrrüsselkäfer (RSH 28). Das Holz eines Baumes wird also seiner Holzart und seinem allmählichen Verfall entsprechend von ganz bestimmten, sich gegenseitig ablösenden Insektenarten befallen, die seine Remineralisierung vorantreiben. Damit schließt sich ein Kreislauf, der den Fortbestand eines Waldes gewährleistet.

Unerwähnt geblieben sind bisher die Tiere, die zwar für die Remineralisierung des Holzes direkt nichts leisten, aber dennoch in der oben dargestellten Besiedlungsfolge eine Rolle spielen. Gemeint sind Prädatoren und Parasiten, von denen auch für das letzte Interglazial je ein Nachweis gelang. Die Spechte sind zu den Prädatoren zu rechnen. Sie meißeln manchmal tiefe Trichter in das Holz, um an die im Holz verborgenen Insektenlarven heranzukommen. Ihre Arbeit ist im Fossilmaterial RSH 21 überliefert. Parasitische Insekten sind besonders innerhalb der Hymenoptera zahlreich vertreten. Eine oder mehrere ihrer Larven entwickeln sich in der Wirtslarve, nachdem die Eier mit Hilfe eines entsprechend langen Legestachels direkt an oder in die Wirtslarve abgelegt wurden. Die nach ihrer Verpuppung schlüpfenden Parasiten nagen sich oftmals durch eigene Gänge nach außen, so daß ein Befall an den in der Regel sehr kleinen Fluglöchern erkannt werden kann (RSH 28). Schließlich können die verlassenen Bohrgänge xylobionter Insektenlarven anderen Insekten als Kinderstube dienen. Unter den hier belegten fossilen Hölzern ist auch dieser Fall in RSH 21 belegt, 
Tab. 1: Übersicht der Arthropoden, die in den Hölzern aus dem letzten Interglazial in der nördlichen Oberrheinebene nachgewiesen wurden. Einteilung in primäre, sekundäre und tertiäre Holzinsekten nach VITE 1952/53.

\begin{tabular}{|c|c|c|c|c|}
\hline Taxon & $\begin{array}{l}\text { Aufschluß der } \\
\text { Zellulose durch }\end{array}$ & Biologische Rolle & Holzart & $\begin{array}{l}\text { Fundort } \\
\text { und Fundnummer }\end{array}$ \\
\hline \multicolumn{5}{|l|}{$\begin{array}{l}\text { INSECTA } \\
\text { Coleoptera }\end{array}$} \\
\hline $\begin{array}{l}\text { Anobium sp. (Anobiidae) } \\
\text { ? Ptilinus sp. (Anobiidae) } \\
\text { Buprestis sp. (Buprestidae) } \\
\text { Tetropium sp. (Cerambycidae) } \\
\text { Callidium sp. (Cerambycidae) } \\
\text { Xyloterus lineatus (Scolytidae) } \\
\text { Cossonina gen. sp. (Curculionidae) }\end{array}$ & $\begin{array}{l}\text { eigene Zellulase } \\
\text { eigene Zellulase } \\
\text { eigene Zellulase } \\
\text { Ectosymbionten } \\
\text { ? } \\
\text { Ectosymbionten } \\
\text { Pilze im Holz }\end{array}$ & $\begin{array}{l}\text { prim.-sek. Holzins. } \\
\text { sek. Holzinsekt } \\
\text { tert. Holzinsekt } \\
\text { sek.-tert. Holzins. } \\
\text { sek.-tert. Holzins. } \\
\text { prim.-sek. Holzins. } \\
\text { tert. Holzinsekten }\end{array}$ & $\begin{array}{l}\text { Fraxinus } \\
\text { Ulmus } \\
\text { Abies } \\
\text { Picea/Larix } \\
\text { Picea/Larix } \\
\text { Abies } \\
\text { Picea/Larix }\end{array}$ & $\begin{array}{l}\text { Geinsheim, RSH } 25 \\
\text { Groß-Rohrheim, RSH } 22 \\
\text { Groß-Rohrheim, RSH } 24 \\
\text { Biblis, RSH } 21 \\
\text { Groß-Rohrheim, RHS } 28 \\
\text { Groß-Rohrheim, RSH } 23 \\
\text { Groß-Rohrheim, RSH } 28\end{array}$ \\
\hline \multicolumn{5}{|l|}{ Hymenoptera } \\
\hline $\begin{array}{l}\text { Paururus juvencus (Siricidae) } \\
\text { Trypoxylon sp./Pison sp. (Sphecidae) } \\
\text { Chalcididae/Bethylidae/Braconidae } \\
\text { gen. sp. }\end{array}$ & $\begin{array}{l}\text { Ectosymbionten } \\
- \\
-\end{array}$ & $\begin{array}{l}\text { sek. Holzinsekt } \\
\text { Wiederbenutzer } \\
\text { eines Ganges } \\
\text { Parasiten }\end{array}$ & $\begin{array}{l}\text { Picea/Larix } \\
\text { Picea/Larix } \\
\text { Picea/Larix }\end{array}$ & $\begin{array}{l}\text { Biblis, RSH } 20 \\
\text { Biblis, RSH } 21 \\
\text { Groß-Rohrheim, RSH } 28\end{array}$ \\
\hline $\begin{array}{l}\text { CHELICERATA } \\
\text { Arachnida }\end{array}$ & & & & \\
\hline Araneus sp. (Araneidae) & - & Beute & Picea/Larix & Biblis, RSH 21 \\
\hline
\end{tabular}


wo die Nutzung einer alten Puppenwiege eines Bockkäfers als Brutkammer einer Grabwespenart anhand der eingetragenen Spinnen nachgewiesen werden konnte.

Obwohl der Fossilbericht stets lückenhaft ist, gibt diese kleine Aufsammlung von fossilen Hölzern aus den Rheinschottern einen Eindruck von der umfangreichen und in vielfacher Hinsicht ineinandergreifenden Biozönose des Waldes. Die Aufschlüsselung ist allerdings nur durch den Vergleich mit rezenten Modellen möglich. In einem natürlichen Wald ist der Kreislauf vom Aufbau des Holzes bis zu dessen vollständiger Remineralisierung geschlossen. Daher haben wir es bei den Hölzern aus dem Rheinschotter mit Sonderfällen zu tun, weil hier einzelne Hölzer in verschiedenen Stadien der Zersetzung aus dem Kreislauf herausgenommen sind. Solche Sonderfälle sind besonders im Bereich von Flußauen zu erwarten, wo entweder der intakte Wald durch Flußerosion angeschnitten wird, oder wo Hochwässer Hölzer aus dem Überschwemmungsbereich dem Ökosystem Wald entnehmen und in den Ablagerungsbereich des Flusses bringen. Erst mit der Einsedimentierung wird das Holz einem weiteren biologischen Abbau entzogen.

\section{Klimatischer Aussagewert der Holzinsekten}

Die Funde von Holzinsekten an interglazialen Hölzern erlauben grundsätzlich mehr als die Feststellung, daß im letzten Interglazial ähnliche Biozönosen existiert haben wie heute. Die Funde können für eine palökologische und paläoklimatologische Ausdeutung von $\mathrm{Be}$ deutung sein. Zwar sind die Holzinsekten an Hölzer gebunden und können keine Klimazonen belegen, die nicht auch durch die Verbreitungsgebiete der entsprechenden Hölzer abgedeckt sind, wie etwa eine glaziale Tundra. Aber innerhalb der Verbreitungsgebiete der Hölzer können stenotherme Arten von Holzinsekten aus klimatischen Gründen einen wesentlich begrenzteren Raum besiedeln, wodurch differenzierte Aussagen möglich werden.

Die bis jetzt aus den Schottern des Oberrheingebietes vorliegenden Taxa kommen alle im heutigen Klima Mitteleuropas vor, was noch keine Einschränkung der Klimaaussage durch die Hölzer bedeutet. Das liegt an dem noch begrenzten Ausschnitt aus dieser Fauna und der noch ungenügenden Bestimmung einzelner Funde.

Stenotherme Arten wurden noch nicht identifiziert. Wegen des Auftretens von Hippopotamus ist aber auch mit warm-stenothermen bzw. atlantischen Faunenelementen zu rechnen. Diese Erwartung wird durch Befunde aus dem entsprechenden Zeitabschnitt Englands, dem Ipswichian, gestützt, in dem ebenfalls Flußpferd und Damhirsch belegt sind. CoOPE (1974) konnte in Käferfaunen des Ipswichian neben einigen nicht näher bestimmten Arten, die er dem mediterranen Raum zuweist, auch einen Kotkäfer (Ontbopagus opacicollis) nachweisen, der heute im mediterranen Raum, aber nicht im etwas kühleren Mittel- und Westeuropa, verbreitet ist.

Im Laufe weiterer Untersuchungen rechnen wir damit, die Zahl der bis zum Artniveau bestimmten Holzinsekten wesentlich zu vergrößern und darauf eine Klimaanalyse zu stützen, die unabhängig die bisherigen Untersuchungen zur Säugetierfauna, zu den Gastropoden, zu den Hölzern und zur Pollenflora ergänzen wird.

\section{Danksagung}

Diese Studie ist im Rahmen des vom BMFT, Bonn, gefördeten Projektes „Terrestrische Paläoklimatologie - Paläoklima am Oberrhein" entstanden. Für die vielseitige Förderung 
danken die Autoren. Besonderer Dank gilt Herrn Prof. K. U. Leistikow, Frankfurt, für die Betreuung von Frau Dipl.-Biol. Kreuz bei der Bestimmung der Hölzer, deren Aufsammlung wir u.a. Herrn Dr. D. Schweiss, Darmstadt, verdanken. Bei der Bestimmung und Bewertung der Arthropodenreste haben uns freundlicherweise die Herren Dr. F. Bachmaier, München; Dr. h.c. S. Cymorek, Krefeld; Dr. C. v. Dehmelt, Klagenfurt, sowie Dr. M. Grasshoff, Frankfurt, mit Rat und Tat zur Seite gestanden. Die REM-Aufnahmen konnten dank des freundlichen Entgegenkommens von Dr. G. Richter am Stereoscan 600 des Senckenbergmuseums, Frankfurt, aufgenommen werden. Die Zeichnungen fertigte Frau I. Lehnen, Darmstadt an und Frau S. Keim übernahm geduldig die Schreibarbeiten.

Herzlichen Dank sagen die Autoren weiterhin den Betriebs- und Baggerführern der Kiesgruben im Oberrheingebiet, ohne deren Hilfe und Verständnis für unsere Sonderwünsche die Bergung des wissenschaftlich bedeutenden Materials nicht möglich gewesen wäre.

\section{Schriftenverzeichnis}

ANONYmus (ohne Jahr): Holzschädlinge I und II. - Desowag-Bayer Holzschutz GmbH, Düsseldorf

ARbeITSGRUPPE (1980): Hydrologische Kartierung und Grundwasserbewirtschaftung, Rhein-NeckarRaum. Analyse des Istzustandes. - Landw. Ministerien Baden Württemberg, Hessen, Rheinland-Pfalz, 71 S.; Stuttgart, Wiesbaden, Mainz.

BARTZ, J. (1976): Quartär und Jungtertiär im Raum Rastatt. - Jb.geol. Landesamt BadenWürttemberg, 18: 121-178; Freiburg i. Br.

BECKER, G. (1950): Bestimmung von Insektenfraßschäden an Nadelholz. - Z. angew. Entomol., 31: 275-303.

Bischoff, H. (1927): Biologie der Hymenopteren. Eine Naturgeschichte der Hautflügler, 698 S.; Berlin.

BRaunS, A. (1962/1966): Taschenbuchführer: Waldinsekten und Streubewohner. - Staatl. Naturhist. Museum z. Braunschweig, $182 \mathrm{~S}$.

- (1964): Taschenbuch der Waldinsekten. - 817 S.; Stuttgart.

BuRGH, J. van der (1978): Hölzer aus dem Pliozän der Niederrheinischen Bucht. - Fortschr. Geol. Rheinld u. Westfl. 28: 213-275; Krefeld.

Burmeister, F. (1964): Coleoptera-Käfer. - in: E. Stresemann: Exkursionsfauna; Berlin.

Coope, G.R. (1969): The response of Coleoptera to gross thermal changes during the Mid Weichselian interstadial. - Mitt. Internat. Verein Limnol., 17: 173-183

- (1974): Interglacial Coleoptera from Bobbitshole, Ipswich, Suffolk. - J. geogr. Soc. Lond., 130: $330-340$.

- (1975): Climatic fluctuations in northwest Europe since the last Interglacial, indicated by fossil assemblages of Coleoptera. - Geol. J. Spec. Iss., 6: 153-168.

- (1977): Fossil coleopteran assemblages as sensitive indicators of climatic changes during the Devensian (last) cold stage. - Phil. Trans. R. Soc. London 280: 313-340.

- (1979): Late cenozoic fossil Coleoptera: Evolution, Biogeography and Ecology. - Ann. Rev. Ecol. Syst., 10: 247-267.

Demelt, C.v. (1966): Die Tierwelt Deutschlands und der angrenzenden Meeresteile, II: Bockkäfer oder Cerambycidae. - 52: 115 S.; Jena.

EsCHERICH, K. (1923): Die Forstinsekten Mitteleuropas. - II: 663 S.; Berlin.

- (1942): Die Forstinsekten Mitteleuropas. - V: Hymenoptera und Diptera, 746 S.; Berlin. 
FIT'SCHEN, J. (1983): Gehölzflora. - 396 S. 651 Abb. Heidelberg (Quelle Meyer).

Friese, H. (1926): Die Insekten Mitteleuropas - I/1 Hymenopteren, Die Bienen, Wespen, Grabund Goldwespen. - 192 S.; Stuttgart.

Greguss, P. (1955): Xylotomische Bestimmung der heute lebenden Gymnospermen. 1-308, 8 Tab., 360 Taf.; Budapest (Akad. Kiado).

HeGI, G. (1958): Illustrierte Flora von Mitteleuropa. 2. Aufl.; Berlin, Hamburg.

HoRION, A. (1951): Verzeichnis der Käfer Mitteleuropas. - I: 536 S., II: 266 S.; Stuttgart.

- (1974): Faunistik der mitteleuopäischen Käfer. - 12: Cerambycidae, 228 s.; Überlingen.

JacoBs, W. \& RenNeR, M. (1974): Taschenlexikon zur Biologie der Insekten. - 635 S.; Stuttgart.

Klausnitzer, B. \& SANDER, F. (1978): Die Bockkäfer Mitteleuropas. — Die Neue Brehm-Bücherei, 222 S.; Wittenberg.

KocH, R. (1910): Bestimmung der Insektenschäden an Fichte und Tanne. - 120 S.; Berlin.

Koenigswald, W.v. (1983): Die Säugetierfauna des süddeutschen Pleistozäns. - in Müller-Beck, H.: Urgeschichte in Baden-Württemberg, 167-216; Stuttgart.

- \& LÖSCHER, M. (1982): Jungpleistozäne Hippopotamus-Funde aus der Oberrheinebene und ihre biogeographische Bedeutung. - N. Jb. Geol. Paläont. Abh., 163: 331-348; Stuttgart.

Meusel, H.; JÄGER, E. \& WeINERT, E. (1965): Vergleichende Chorologie der zentraleuropäischen Flora. - Jena.

Mourier, H. \& Winding, O. (1975): Collins Guide to Wild Life in House and Home. - 224 S.; London.

Roselt, G. \& Feustel, H. (1960): Ein Taxodiazeenholz aus der Mitteldeutschen Braunkohle mit Insektenspuren und -resten. - Geologie, 9/1: 84-101; Berlin.

SCHEER. H.D. (1978): Gliederung und Aufbau der Niederterrassen von Rhein und Main im nördlichen Oberrheingraben - Geol. Jb. Hessen, 106: 273-289; Wiesbaden.

Schweingruber, F.H. (1978): Mikroskopische Holzanatomie. - 240 S., 275 Abb., Zug (Haupt).

Vite, J.P. (1952/1953): Die holzzerstörenden Insekten Mitteleuropas. - Textb. 155 S., Tafelb. 78 S.; Göttingen (Musterschmidt).

Weber, H. \& WeIDNER, H. (1974): Grundriß der Insektenkunde. - 5. Aufl.; Stuttgart.

Welten, M. (1981): Verdrängung und Vernichtung der anspruchsvollen Gehölze am Beginn der letzten Eiszeit und die Korrelation der Frühwürm-Interstadiale in Mittel- und Nordeuropa. Eiszeitalter u. Gegenwart, 31: 187-202; Hannover. 
154 Ralf Klinger \& Wighart von Koenigswald mit einem Beitrag von Angela Kreuz 